\section{Sternenkatarakt}

Wegen eines zunehmenden einseitigen Sehverlusts im Verlauf der letzten sechs Monate wurde ein 55-jähriger Mann von seinem Hausarzt zum Augenarzt überwiesen.

Der Patient berichtete, er habe neun Monate zuvor einen Faustschlag auf dieses Auge bekommen. Bei der Untersuchung erkannte man eine sternförmige Trübung der Linse. Es handelt sich um eine traumatische Katarakt, die durch eine stumpfe oder penetrierende Augenverletzung zustande kommt. Als hypothetischer

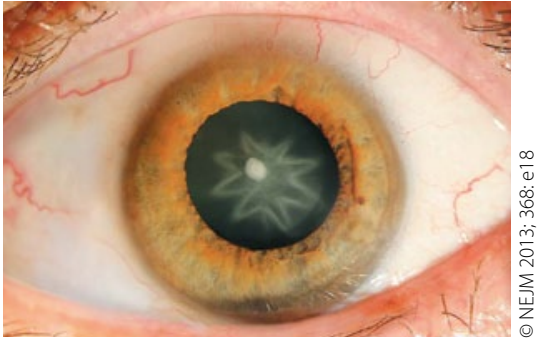

Katarakt nach stumpfem Trauma.

Mechanismus für diese indirekte Verletzung wird eine durch das Auge laufende Schockwelle angenommen. Die Verlet- zung der Linse kann zu einer stern- oder rosettenförmigen Katarakt führen.

Wenn sich derartige Trübungen im Bereich der optischen Achse befinden, ist eine Katarakt-Operation erforderlich. Der Patient erlangte seine volle Sehkraft wieder nach einer erfolgreichen Phakoemulsifikation und Implantation einer intraokulären Linse.

H. S. FÜESSL =

- C. Zehetner und N. Bechrakis (claus.zehetner@i-med.ac.at): Stellate cataract. New Engl. J. Med. 2013; 368: e18

\title{
Typ-1-Diabetiker: Cave Gewichtszunahme
}

\section{Unter einer intensiven Insulinthera- pie nehmen Diabetiker oft erheblich an Gewicht zu. Nun liegt erstmals eine kontrollierte Studie zur Ent- wicklung einer subklinischen Arterio- sklerose bei diesen Patienten vor.}

- In der Epidemiology of Diabetes Interventions and Complications (EDIC) Study, in der nach Beendigung der DCCT-Studie 1168 Typ-1-Diabetiker im Alter von über 18 Jahren betreut werden, untersuchte man das Arterioskleroserisiko und klinische Endpunkte der Studienteilnehmer. Die Patienten wurden in solche mit konventioneller Insulintherapie (ein bis zwei Injektionen/ Tag) und solche mit intensiver Insulintherapie (drei oder mehr Injektionen / Tag) randomisiert. Nach einem Jahr und nach sechs Jahren wurde die IntimaMedia-Dicke (IMT) und nach acht Jahren ein koronararterieller KalziumScore ermittelt. Die Zunahme des BodyMass-Index (BMI) und des Taillenumfangs unter der intensiven Insulintherapie wurde in Quartile eingeteilt. Patienten mit exzessiver Gewichtszunahme (im Mittel ca. sieben BMI-Einheiten) hatten im Vergleich zu Patienten mit geringer Gewichtszunahme (im Mittel we-

niger als drei BMI-Einheiten) nach einem Jahr und nach sechs Jahren um 0,6\% bzw. 1,1\% höhere $\mathrm{HbA}_{1 c}$-Werte trotz einer etwas höheren Insulindosis.

Unter der konventionellen Insulintherapie nahm im gleichen Zeitraum bei Patienten mit exzessiver Gewichtszunahme der $\mathrm{HbA}_{1 \mathrm{c}}$-Wert um $0,5 \%$ ab. Unter einer intensiven Insulintherapie verschlechterten sich bei Patienten mit exzessiver Gewichtszunahme Komponenten des metabolischen Syndroms wie Taillenumfang, Blutdruck und Tri-

\section{Kommentar}

Die Studie zeigt, dass sich bei exzessiver Gewichtszunahme unter einer intensiven Insulintherapie eine abdominale Adipositas und eine Insulinresistenz einstellt; Blutdruck sowie Lipide verschlechtern sich. Auch Komponenten des metabolischen Syndroms nehmen zu. Die größte IMT und der höchste Kalzium-Score wurden bei Personen mit starker Gewichtszunahme festgestellt. Eine intensive Insulintherapie reduziert bei Typ-1-Diabetikern nicht nur mikrovaskuläre Komplikationen, sondern auch - im Unterschied zu Typ-2-Diabetikern - makrovaskuläre Folgekrankheiten. Die vorliegende Studie zeigt nun erstmals, dass eine subklinische glyzeride. Das HDL-Cholesterin stieg jedoch aus nicht erklärbaren Gründen an. Je deutlicher der Gewichtsanstieg in einem Jahr bzw. sechs Jahren war, desto größer war auch die IMT an den Karotiden.

Die IMT korrelierte mit traditionellen kardiovaskulären Risikofaktoren. Ähnlich wie die IMT war auch der Kalzium-Score unter einer intensiven Insulintherapie bei den Personen am höchsten, die am meisten Gewicht zugenommen hatten. 